\title{
Generalized Jordan triple derivations associated with Hochschild 2-cocycles of rings
}

\author{
O. H. Ezzat ${ }^{*}$ and H. Nabiel
}

*Correspondence:
ohezzat@azhar.edu.eg
Department of Mathematics,
Faculty of Science, Al-Azhar
University, Nasr City, Cairo, 11884,
Egypt

Egypt

\begin{abstract}
In the present work, we introduce the notion of a generalized Jordan triple derivation associated with a Hochschild 2-cocycle, and we prove results which imply under some conditions that every generalized Jordan triple derivation associated with a Hochschild 2 -cocycle of a prime ring with characteristic different from 2 is a generalized derivation associated with a Hochschild 2-cocycle.
\end{abstract}

Keywords: Prime ring, Derivation, Generalized Jordan triple derivation, Hochschild 2-cocycle

\section{Introduction}

Let $R$ denote an associative ring with center $Z(R)$. A ring $R$ is said to have characteristic $n$ if $n$ is the least positive integer such that $n x=0$ for all $x \in R$, and of characteristic not $n$ if $n x=0, x \in R$, then $x=0$. An additive subgroup $L$ of $R$ is called a Lie ideal of $R$ if $[u, r] \in L$ for all $u \in L, r \in R$. A Lie ideal $L$ is said to be a square-closed Lie ideal of $R$ if $u^{2} \in L$ for all $u \in L$. An $R$-bimodule $M$ is a left and right $R$-module such that $x(m y)=(x m) y$ for all $m \in M$ and $x, y \in R$. Recall that a ring $R$ is called prime if $x R y=(0)$ implies that either $x=0$ or $y=0$, and $R$ is called semiprime if $x R x=(0)$ implies $x=0$. An additive mapping $d: R \rightarrow R$ is called a derivation if $d(x y)=d(x) y+x d(y)$ for all $x, y \in R . d$ is called a Jordan derivation in case $d\left(x^{2}\right)=d(x) x+x d(x)$ for all $x \in R$. Moreover, $d$ is called a Jordan triple derivation if $d(x y x)=d(x) y x+x d(y) x+x y d(x)$ for all $x, y \in R$. It is obvious to see that every derivation is a Jordan derivation and is a Jordan triple derivation but the converse is in general not true. A classical result of Herstein [1] asserts that any Jordan derivation of a prime ring with characteristic different from 2 is a derivation. In [2], Brešar has proved Herstein's result in the case of a semiprime ring. Also, he has shown in [3] that any Jordan triple derivation of a 2-torsion free semiprime ring is a derivation. An additive map $f$ of a ring $R$ is called a generalized derivation if there is a derivation $d$ of $R$ such that for all $x, y$ in $R, f(x y)=f(x) y+x d(y)$ and is called a generalized Jordan derivation if there is a Jordan derivation $d$ such that $f\left(x^{2}\right)=f(x) x+x d(x)$ for all $x \in R$. Furthermore, $f$ is said to be a generalized Jordan triple derivation if there is a Jordan triple derivation $d$ of $R$ such that for all $x, y$ in $R, f(x y x)=f(x) y x+x d(y) x+x y d(x)$. In [4], Jing and Lu have proved in a prime ring $R$ of characteristic not two that every generalized Jordan derivation of $R$ 
is a generalized derivation, and also every generalized Jordan triple derivation on $R$ is a generalized derivation.

Let $\theta$ and $\phi$ be endomorphisms of a ring R.f is called a $(\theta, \phi)$-derivation if $f(x y)=$ $f(x) \theta(y)+\phi(x) f(y)$ for all $x, y \in R . f$ is called a Jordan $(\theta, \phi)$-derivation if $f\left(x^{2}\right)=$ $f(x) \theta(x)+\phi(x) f(x)$ for all $x \in R$. $f$ is called a Jordan triple $(\theta, \phi)$-derivation if $f(x y x)=$ $f(x) \theta(y) \theta(x)+\phi(x) f(y) \theta(x)+\phi(x) \phi(y) f(x)$ for all $x, y \in R$. In [5], Liu and Shiue have proved that every Jordan triple $(\theta, \phi)$-derivation on a 2-torsion free semiprime ring $R$ is a $(\theta, \phi)$-derivation, where $\theta$ and $\phi$ are automorphisms. An additive mapping $f: R \rightarrow R$ is said to be a left (right) centralizer, if $f(x y)=f(x) y(f(x y)=x f(y))$ for all $x, y \in R$. $f$ is called a centralizer, if $f$ is both a left and right centralizer. In [6], Vukman and Kosi-Ulbl have shown that if $R$ is a 2-torsion free semiprime ring and $f$ is an additive mapping of $R$ such that $2 f(x y x)=f(x) y x+x y f(x)$ for all $x, y \in R$, then $f$ is a centralizer.

An additive mapping $f: R \rightarrow R$ is said to be a left (right) $\theta$-centralizer associated with a function $\theta$ of $R$, if $f(x y)=f(x) \theta(y)(f(x y)=\theta(x) f(y))$ for all $x, y \in R . f$ is called a $\theta$-centralizer, if $f$ is both a left and right $\theta$-centralizer. Daif, El-Sayiad, and Muthana in [7] have proved that if $R$ is a 2-torsion free semiprime ring and $f$ is an additive mapping of $R$ such that $2 f(x y x)=f(x) \theta(y x)+\theta(x y) f(x)$ for all $x, y \in R$ with $\theta(Z(R))=Z(R)$, where $\theta$ is a nonzero surjective endomorphism on $R$, then $f$ is a $\theta$-centralizer.

Now let $R$ be a ring and $M$ be an $R$-bimodule. A biadditive map $\alpha: R \times R \rightarrow M$ is called a Hochschild 2-cocycle, if $x \alpha(y, z)-\alpha(x y, z)+\alpha(x, y z)-\alpha(x, y) z=0$ for all $x, y, z \in R$, and $\alpha$ is called symmetric if $\alpha(x, y)=\alpha(y, x)$ for all $x, y \in R$. Nakajima [8] has introduced a new type of generalized derivations and generalized Jordan derivations associated with Hochschild 2-cocycles in the following way. An additive map $f: R \rightarrow M$ is called a generalized derivation associated with a Hochschild 2-cocycle $\alpha$ if $f(x y)=$ $f(x) y+x f(y)+\alpha(x, y)$ for all $x, y \in R$, and $f$ is called a generalized Jordan derivation associated with $\alpha$ if $f\left(x^{2}\right)=f(x) x+x f(x)+\alpha(x, x)$ for all $x \in R$. If $\alpha=0$, then $f$ means the usual derivation and Jordan derivation. He has given the following examples:

(1) If $f$ is a generalized derivation associated with a derivation $d$, then the map $\alpha_{1}$ : $R \times R \ni(x, y) \mapsto x(d-f)(y) \in M$ is biadditive and satisfies the 2-cocycle condition. Hence, $f$ is a generalized derivation associated with $\alpha_{1}$.

(2) If $f: R \rightarrow M$ is a left centralizer, then by $f(x y)=f(x) y+x f(y)+x(-f)(y)$, we have a 2-cocycle $\alpha_{2}: R \times R \rightarrow M$ defined by, $\alpha_{2}(x, y)=x(-f)(y)$, and hence, $f$ is a generalized derivation associated with $\alpha_{2}$.

(3) Let $f$ be a $(\theta, \phi)$-derivation. Then, the map $\alpha_{3}: R \times R \ni(x, y) \mapsto f(x)(\theta(y)-y)+$ $(\phi(x)-x) f(y) \in M$, is biadditive and satisfies the 2-cocycle condition. Since $f(x y)=$ $f(x) y+x f(y)+\alpha_{3}(x, y)$, then $f$ is a generalized derivation associated with $\alpha_{3}$.

(4) In general, he has mentioned the following. Let $f: R \rightarrow M$ be an additive map and let $\alpha: R \times R \rightarrow M$ be a biadditive map. If $f(x y)=f(x) y+x f(y)+\alpha(x, y)$ holds, then by the associativity $f((x y) z)=f(x(y z)), \alpha$ satisfies the 2 -cocycle condition. Thus $f$ is a generalized derivation associated with $\alpha$.

In his work, Nakajima [8] has shown the following result. Let $R$ be a 2-torsion free ring. Then, every generalized Jordan derivation associated with a Hochschild 2-cocycle $\alpha$ is a generalized derivation associated with $\alpha$ in each of the following cases:

(i) $R$ is a noncommutative prime ring.

(ii) There exist $x, y \in R$ such that $[x, y]$ is a nonzero divisor.

(iii) $R$ is commutative and $\alpha$ is symmetric. 
Nawzad, et al. [9] have shown the following. Let $R$ be a 2-torsion free ring. Then, every generalized Jordan derivation associated with a Hochschild 2-cocycle $\alpha$ is a generalized derivation associated with $\alpha$ in each of the following cases:

(i) $R$ is a noncommutative semiprime ring and $\alpha$ is symmetric.

(ii) $R$ is commutative.

In [10], Rehman and Hongan have proved the following result. Let $R$ be a 2-torsion free ring and $L$ a square-closed Lie ideal of $R$. Then, every generalized Jordan derivation associated with a Hochschild 2-cocycle $\alpha$ is a generalized derivation associated with $\alpha$ in each of the following cases.

(i) $R$ is a prime ring and $L$ is noncommutative.

(ii) $R$ is a prime ring, $L$ is commutative and $\alpha$ is symmetric.

(iii) There exist $x, y \in R$ such that $[x, y]$ is a nonzero divisor in $L$.

In the present article, we introduce the notion of generalized Jordan triple derivations associated with Hochschild 2-cocycles in the following way. Let $R$ be a ring and let $M$ be an $R$-bimodule. An additive $\operatorname{map} f: R \rightarrow M$ is called a generalized Jordan triple derivation associated with a Hochschild 2-cocycle $\alpha$ if $f(x y x)=f(x) y x+x f(y) x+\alpha(x, y) x+x y f(x)+$ $\alpha(x y, x)$ for all $x, y \in R$.

Examples (i) If $f$ is a Jordan triple derivation, then the zero map $\alpha_{1}$ is biadditive and satisfies the 2 -cocycle condition. Therefore $f$ is a generalized Jordan triple derivation associated with $\alpha_{1}$.

(ii) If $f$ is a generalized Jordan triple derivation associated with a Jordan triple derivation $d$, then $\alpha_{2}(x, y)=x(d-f)(y)$ is biadditive and satisfies the 2-cocycle condition and we can see that $f(x y x)=f(x) y x+x f(y) x+\alpha_{2}(x, y) x+x y f(x)+\alpha_{2}(x y, x)$. Hence $f$ is a generalized Jordan triple derivation associated with $\alpha_{2}$.

Our aim in this work is to show that every generalized Jordan triple derivation associated with a Hochschild 2-cocycle $\alpha$ from a prime ring $R$ with characteristic different from 2 to an $R$-bimodule $M$ is a generalized derivation associated with $\alpha$.

\section{Preliminary results}

The proof of our result is based on the following series of auxiliary lemmas.

Lemma 1 Let $f$ be a generalized Jordan triple derivation from a ring $R$ to an $R$-bimodule $M$ associated with a Hochschild 2-cocycle map $\alpha$ from $R \times R$ into $M$. Then for all $x, y, z \in$ $R, f(x y z+z y x)=f(x) y z+x f(y) z+\alpha(x, y) z+z y f(z)+\alpha(x y, z)+f(z) y x+z f(y) x+\alpha(z, y) x+$ $z y f(x)+\alpha(z y, x)$.

Proof Let $v=f((x+z) y(x+z))$, we have for all $x, y, z \in R$

$$
\begin{aligned}
0= & v-v \\
= & f(x y x)+f(x y z+z y x)+f(z y z)-\{f(x+z) y(x+z) \\
& +(x+z) f(y)(x+z)+\alpha(x+z, y)(x+z) \\
& +(x+z) y f(x+z) \\
& +\alpha((x+z) y,(x+z))\} .
\end{aligned}
$$

Then, 


$$
\begin{aligned}
0= & f(x y x)+f(x y z+z y x)+f(z y z)-\{f(x) y x \\
& +f(x) y z+f(z) y x \\
+ & f(z) y z+x f(y) x+x f(y) z+z f(y) x+z f(y) z \\
& +\alpha(x, y) x+\alpha(x, y) z \\
+ & \alpha(z, y) x+\alpha(z, y) z+x y f(x)+x y f(z)+z y f(x) \\
& +z y f(z)+\alpha(x y, x) \\
+ & \alpha(x y, z)+\alpha(z y, x)+\alpha(z y, z)\} \text { for all } x, y, z \in R,
\end{aligned}
$$

Therefore,

$$
\begin{aligned}
f(x y z+z y x)= & f(x) y z+x f(y) z+\alpha(x, y) z+z y f(z) \\
& +\alpha(x y, z) \\
& +f(z) y x+z f(y) x+\alpha(z, y) x+z y f(x) \\
& +\alpha(z y, x) \text { for all } x, y, z \in R,
\end{aligned}
$$

as required.

For a generalized Jordan triple derivation $f$ from a $\operatorname{ring} R$ to an $R$-bimodule $M$ associated with a Hochschild 2-cocycle $\alpha$, we denote by $\delta, F$ and $\beta$ the maps from $R \times R \times R$ into $M$ defined by $\delta(x, y, z)=f(x y z)-f(x) y z-x f(y) z-\alpha(x, y) z-x y f(z)-\alpha(x y, z), F(x, y, z)=$ $f(x y z)-f(x) y z-x f(y) z-x y f(z)$ and $\beta(x, y, z)=x y z-z y x$, respectively. Thus, $\delta(x, y, z)=$ $F(x, y, z)-\alpha(x, y) z-\alpha(x y, z)$.

Lemma 2 For all $x, y, z$ in a ring $R$, the following hold:

(i) $\delta(x, y, z)=-\delta(z, y, x)$, and

(ii) $\delta(x, y, z)$ and $\beta(x, y, z)$ are tri-additive.

Proof (i) Follows easily from Lemma 1.

(ii) Replace $x$ by $a+b$ in the definition of $\delta$, then (ii) is easily seen.

Lemma 3 For any ring $R$ and any $a, b, c, x \in R$,

$\delta(a, b, c) x \beta(a, b, c)+\beta(a, b, c) x \delta(a, b, c)=0$.

Proof Let $v=f(a b c x c b a+c b a x a b c)$, then $0=v-v=f((a b c) x(c b a)+(c b a) x(a b c))-$ $f(a(b c x c b) a+c(b a x a b) c)$. By the definition of the generalized Jordan triple derivation $f$ associated with a Hochschild 2-cocycle $\alpha$ and by Lemma 1, we get

$$
\begin{aligned}
0 & =f(a b c) x c b a+a b c f(x) c b a+\alpha(a b c, x) c b a+a b c x f(c b a) \\
& +\alpha(a b c x, c b a)+f(c b a) x a b c+c b a f(x) a b c+\alpha(c b a, x) a b c \\
& +c b a x f(a b c)+\alpha(c b a x, a b c)-\{f(a) b c x c b a+a f(b) c x c b a \\
& +a b f(c) x c b a+a b c f(x) c b a+a b \alpha(c, x) c b a+a b c x f(c) b a \\
& +a b \alpha(c x, c) b a+a \alpha(b, c x c) b a+a b c x c f(b) a+a \alpha(b c x c, b) a \\
& +\alpha(a, b c x c b) a+a b c x c b f(a)+\alpha(a b c x c b, a)+f(c) b a x a b c \\
& +c f(b) a x a b c+c b f(a) x a b c+c b a f(x) a b c+c b \alpha(a, x) a b c \\
& +c b a x f(a) b c+c b \alpha(a x, a) b c+c \alpha(b, a x a) b c+c b a x a f(b) c \\
& +c \alpha(b a x a, b) c+\alpha(c, b a x a b) c+c b a x a b f(c)+\alpha(c b a x a b, c)\} .
\end{aligned}
$$


Therefore, for all $a, b, c, x \in R$

$$
\begin{aligned}
0 & =F(a, b, c) x c b a+a b c x F(c, b, a) \\
& +\{\alpha(a b c, x)-a b \alpha(c, x)\} c b a+\{\alpha(a b c x, c b a)-\alpha(a b c x c b, a)\} \\
& -\{a b \alpha(c x, c) b a+a \alpha(b, c x c) b a+a \alpha(b c x c, b) a+\alpha(a, b c x c b) a\} \\
& +F(c, b, a) x a b c+c b a x F(a, b, c) \\
& +\{\alpha(c b a, x)-c b \alpha(a, x)\} a b c+\{\alpha(c b a x, a b c)-\alpha(\operatorname{cbaxab}, c)\} \\
& -\{c b \alpha(a x, a) b c+c \alpha(b, a x a) b c+c \alpha(b a x a, b) c+\alpha(c, b a x a b) c\}
\end{aligned}
$$

Since $\alpha$ is a 2-cocycle map, we obtain the following relations for all $a, b, c, x \in R$ :

(i) $\quad\{\alpha((a b) c, x)-(a b) \alpha(c, x)\} c b a=\{\alpha(a b, c x)-\alpha(a b, c) x\} c b a$.

(ii) $\quad \alpha(a b c x,(c b) a)-\alpha((a b c x)(c b), a)=\alpha(a b c x, c b) a-(a b c x) \alpha(c b, a)$.

(iii) $\quad\{\alpha((c b) a, x)-(c b) \alpha(a, x)\} a b c=\{\alpha(c b, a x)-\alpha(c b, a) x\} a b c$.

(iv) $\alpha(\operatorname{cbax},(a b) c)-\alpha((\operatorname{cbax})(a b), c)=\alpha(\operatorname{cbax}, a b) c-(\operatorname{cbax}) \alpha(a b, c)$.

Substituting from (i-iv) in (2), we get for all $a, b, c, x \in R$

$$
\begin{aligned}
0 & =F(a, b, c) x c b a+a b c x F(c, b, a) \\
& +\{\alpha(a b, c x)-\alpha(a b, c) x\} c b a+\{\alpha(a b c x, c b) a-a b c x \alpha(c b, a)\} \\
& -\{a b \alpha(c x, c) b a+a \alpha(b, c x c) b a+a \alpha(b c x c, b) a+\alpha(a, b c x c b) a\} \\
& +F(c, b, a) x a b c+c b a x F(a, b, c) \\
& +\{\alpha(c b, a x)-\alpha(c b, a) x\} a b c+\{\alpha(c b a x, a b) c-\operatorname{cbax} \alpha(a b, c)\} \\
& -\{c b \alpha(a x, a) b c+c \alpha(b, a x a) b c+c \alpha(b a x a, b) c+\alpha(c, b a x a b) c\}
\end{aligned}
$$

Since $\alpha$ is a 2-cocycle map, we conclude for all $a, b, c, x \in R$ that

(i) $\alpha(a b, c x)=a \alpha(b, c x)+\alpha(a, b(c x))-\alpha(a, b)(c x)$.

(ii) $\alpha(a b c x, c b) a=\{-(a b c x) \alpha(c, b)+\alpha((a b c x) c, b)+\alpha(a b c x, c) b\} a$.

(iii) $\alpha(c b, a x)=c \alpha(b, a x)+\alpha(c, b(a x))-\alpha(c, b)(a x)$.

(iv) $\alpha(\operatorname{cbax}, a b) c=\{-(\operatorname{cbax}) \alpha(a, b)+\alpha((\operatorname{cbax}) a, b)+\alpha(\operatorname{cbax}, a) b\} c$.

Substituting from (i-iv) in (3), we obtain

$$
\begin{aligned}
0 & =\{F(a, b, c)-\alpha(a b, c)-\alpha(a, b) c\} x c b a+a b c x\{F(c, b, a)-\alpha(c b, a) \\
& -\alpha(c, b) a\}+\{a \alpha(b, c x) c b a-a b \alpha(c x, c) b a-a \alpha(b, c x c) b a\} \\
& +\{\alpha(a b c x c, b) a-a \alpha(b c x c, b) a-\alpha(a, b c x c b) a\} \\
& +\alpha(a, b c x) c b a+\alpha(a b c x, c) b a+\{F(c, b, a)-\alpha(c b, a) \\
& -\alpha(c, b) a\} x a b c+c b a x\{F(a, b, c)-\alpha(a b, c)-\alpha(a, b) c\} \\
& +\{c \alpha(b, a x) a b c-c b \alpha(a x, a) b c-c \alpha(b, a x a) b c\} \\
& +\{\alpha(c b a x a, b) c-c \alpha(b a x a, b) c-\alpha(c, b a x a b) c\} \\
& +\alpha(c, b a x) a b c+\alpha(c b a x, a) b c, \text { for all } a, b, c, x \in R .
\end{aligned}
$$

Again since $\alpha$ is a 2-cocycle map, we have

(i) $\quad a\{\alpha(b, c x) c-b \alpha(c x, c)-\alpha(b,(c x) c)\} b a=-a \alpha(b(c x), c) b a$.

(ii) $\{\alpha(a(b c x c), b)-a \alpha(b c x c, b)-\alpha(a,(b c x c) b)\} a=-\alpha(a, b c x c) b a$.

(iii) $\quad c\{\alpha(b, a x) a-b \alpha(a x, a)-\alpha(b,(a x) a)\} b c=-c \alpha(b(a x), a) b c$.

(iv) $\{\alpha(c($ baxa $), b)-c \alpha($ baxa,$b)-\alpha(c,($ baxa $) b)\} c=-\alpha(c, b a x a) b c$. 
Replacing (i-iv) into (4), we get, for all $a, b, c, x \in R$

$$
\begin{aligned}
0 & =\delta(a, b, c) x c b a+a b c x \delta(c, b, a)-a \alpha(b c x, c) b a-\alpha(a, b c x c) b a \\
& +\alpha(a, b c x) c b a+\alpha(a b c x, c) b a+\delta(c, b, a) x a b c+c b a x \delta(a, b, c) \\
& -c \alpha(b a x, a) b c-\alpha(c, b a x a) b c+\alpha(c, b a x) a b c+\alpha(\operatorname{cbax}, a) b c .
\end{aligned}
$$

Continuing in this manner, we obtain

(i) $\quad\{-a \alpha(b c x, c)-\alpha(a,(b c x) c)+\alpha(a, b c x) c+\alpha(a(b c x), c)\} b a=0$.

(ii) $\quad\{-c \alpha(b a x, a)-\alpha(c,(b a x) a)+\alpha(c, b a x) a+\alpha(c(b a x), a)\} b c=0$.

By (5), we conclude that $0=\delta(a, b, c) x c b a+a b c x \delta(c, b, a)+\delta(c, b, a) x a b c+c b a x \delta(a, b, c)$ for all $a, b, c, x \in R$. By Lemma 2 , we obtain $0=\delta(a, b, c) x c b a-a b c x \delta(a, b, c)-$ $\delta(a, b, c) x a b c+\operatorname{cbax} \delta(a, b, c)$ for all $a, b, c, x \in R$.

Therefore, $\delta(a, b, c) x \beta(a, b, c)+\beta(a, b, c) x \delta(a, b, c)=0$ for all $a, b, c, x \in R$. This finishes the proof of the lemma.

Lemma 4 If $R$ is a prime ring of characteristic not 2, then for all $a, b, c, x \in$ $R, \delta(a, b, c) x \beta(a, b, c)=0$.

Proof By Lemma 3 and Lemma 1.1 of Brešar [3], we get the proof.

Lemma 5 If $R$ is a prime ring of characteristic not 2, then

$\delta\left(a_{1}, b_{1}, c_{1}\right) x \beta\left(a_{2}, b_{2}, c_{2}\right)=0$ for all $a_{1}, b_{1}, c_{1}, a_{2}, b_{2}, c_{2}, x \in R$

Proof From Lemma 2(ii), Lemma 4, and Lemma 1.2 of Brešar [3], we get the proof.

Lemma 6 Let $R$ be a prime ring. Then, $R$ is commutative iff $\beta(a, b, c)=0$ for all $a, b, c \in R$.

Proof If $R$ is commutative, then, by definition of $\beta, \beta(a, b, c)=0$ for all $a, b, c \in R$. Conversely, assume that $\beta(a, b, c)=0$ for all $a, b, c \in R$. Let $Q$ be the Martindale right ring of quotients of $R$ defined by Martindale [11]. Then $Q$ is a prime ring with identity that contains the ring $R$. By Chuang [12], $Q$ satisfies the same generalized polynomial identities as $R$. In particular $a b c-c b a=0$ for all $a, b, c \in Q$. Replacing $c$ by the identity of $Q$ yields the commutativity of $Q$, and hence $R$.

Lemma 7 Let $R$ be a prime ring of characteristic not 2 . Then $\delta(a, b, c)=0$ for all $a, b, c \in$ $R$, in each of the following cases:

(i) $R$ is noncommutative.

ii There exist $x, y, z \in R$ such that $\beta(x, y, z)$ is a nonzero divisor in $M$.

iii $R$ is commutative and $\alpha$ is symmetric.

Proof (i) By Lemmas 5 and 6, we get our requirement.

(ii) By Lemma 5, we have $\delta(a, b, c) r \beta(x, y, z)=0$ for all $a, b, c, r, x, y, z \in R$. From our assumption $\delta(a, b, c) r=0$ for all $a, b, c, r \in R$. Thus the primeness of $R$ gives $\delta(a, b, c)=$ 0 for all $a, b, c \in R$.

(iii) From Lemma 1 we have $f(a b c+c b a)=f(a) b c+a f(b) c+\alpha(a, b) c+a b f(c)+$ $\alpha(a b, c)+f(c) b a+c f(b) a+\alpha(c, b) a+c b f(a)+\alpha(c b, a)$ for all $a, b, c \in R$. Since $R$ is commutative and $\alpha$ is symmetric, we get $0=2\{f(a b c)-f(a) b c-a f(b) c-a b f(c)\}-$ 
$\alpha(a, b) c-\alpha(a b, c)-a \alpha(b, c)-\alpha(a, b c)$ for all $a, b, c \in R$. Since $\alpha$ is 2-cocycle we have $-a \alpha(b, c)-\alpha(a, b c)=-\alpha(a, b) c-\alpha(a b, c)$ for all $a, b, c \in R$. Therefore $0=2\{f(a b c)-$ $f(a) b c-a f(b) c-a b f(c)-\alpha(a, b) c-\alpha(a b, c)\}$ for all $a, b, c \in R$. Since $R$ has characteristic not 2 , then $\delta(a, b, c)=0$ for all $a, b, c \in R$, as required.

\section{Main result}

Theorem 1 Let $R$ be a prime ring of characteristic not 2. Then every generalized Jordan triple derivation associated with a Hochschild 2-cocycle $\alpha$ is a generalized derivation associated with $\alpha$ in each of the following cases.

(i) $R$ is noncommutative.

(ii) There exist $x, y, z \in R$ such that $\beta(x, y, z)$ is a nonzero divisor in $M$.

(iii) $R$ is commutative and $\alpha$ is symmetric.

Proof Suppose that $f$ is a generalized Jordan triple derivation associated with a Hochschild 2-cocycle $\alpha$. We denote by $G(a, b)$ and $a^{b}$ the elements of $M$ defined by $G(a, b)=f(a b)-f(a) b-a f(b)$, and $a^{b}=f(a b)-f(a) b-a f(b)-\alpha(a, b)$, respectively. Thus, $a^{b}=G(a, b)-\alpha(a, b)$. It is evident that $a^{b+c}=a^{b}+a^{c}$, and $(a+b)^{c}=a^{c}+b^{c}$. By Lemma 7, we have $\delta(a, b, c)=0$ for all $a, b, c \in R$. Thus, for all $a, b, c \in R$

$$
f(a b c)=f(a) b c+a f(b) c+\alpha(a, b) c+a b f(c)+\alpha(a b, c) .
$$

Now let $v=f(a b x a b)$, then $0=v-v=f((a b) x(a b))-f(a(b x a) b)$. By (6), we have for all $a, b, x \in R$

$$
\begin{aligned}
0 & =f(a b) x a b+a b f(x) a b+\alpha(a b, x) a b+a b x f(a b)+\alpha(a b x, a b) \\
& -f(a) b x a b-a f(b) x a b-a b f(x) a b-a \alpha(b, x) a b-a b x f(a) b \\
& -a \alpha(b x, a) b-\alpha(a, b x a) b-a b x a f(b)-\alpha(a b x a, b) .
\end{aligned}
$$

So, for all $a, b, x \in R$

$$
\begin{aligned}
0 & =G(a, b) x a b+a b x G(a, b)+\{\alpha(a b, x)-a \alpha(b, x)\} a b \\
& +\{\alpha(a b x, a b)-\alpha(a b x a, b)\}-a \alpha(b x, a) b-\alpha(a, b x a) b .
\end{aligned}
$$

Since $\alpha$ is 2-cocycle we have for all $a, b, x \in R$ that

(i) $\quad\{\alpha(a b, x)-a \alpha(b, x)\} a b=\{\alpha(a, b x)-\alpha(a, b) x\} a b$, and

(ii) $\quad \alpha(a b x, a b)-\alpha((a b x) a, b)=\alpha(a b x, a) b-(a b x) \alpha(a, b)$.

Substituting from (i) and (ii) in (7), we get $G(a, b) x a b-\alpha(a, b) x a b+a b x G(a, b)-$ $a b x \alpha(a, b)+\alpha(a, b x) a b+\alpha(a b x, a) b-a \alpha(b x, a) b-\alpha(a, b x a) b=0$ for all $a, b, x \in R$. But $\alpha$ is 2-cocycle, hence $\{\alpha(a, b x) a+\alpha(a b x, a)-a \alpha(b x, a)-\alpha(a, b x a)\} b=0$. Therefore $a^{b} x(a b)+(a b) x a^{b}=0$ for all $a, b, x \in R$. By Lemma 1.1 of Brešar [3] , we get

$$
a^{b} x(a b)=(a b) x a^{b}=0 \text { for all } a, b, x \in R .
$$

Replacing $a$ by $a+c$ in (8) and using (8), we obtain $a^{b} x c b=-c^{b} x a b$ for all $a, b, c, x \in R$, and then $\left(a^{b} x c b\right) y\left(a^{b} x c b\right)=-a^{b} x\left(c b y c^{b}\right) x a b=0$ for all $a, b, c, x, y \in R$. Thus the primeness of $R$ gives

$$
a^{b} x c b=0 \text { for all } a, b, c, x \in R .
$$

Similarly replacing $b$ by $b+d$ in (9), we get

$$
a^{b} x c d=0 \text { for all } a, b, c, d, x \in R \text {. }
$$


Putting $c=a^{b}$ and $x=d x$ in (10) we have $a^{b} d x a^{b} d=0$ for all $a, b, d, x \in R$. Again, the primeness of $R$ yields that $a^{b} d=0$ for all $a, b, d \in R$, and hence $a^{b}=0$ for all $a, b \in R$. Consequently, $f$ is a generalized derivation associated with a Hochschild 2-cocycle $\alpha$.

Acknowledgements

The authors are very grateful to Prof. M. N. Daif for his helpful comments and suggestions. This paper is a part of the second author's Ph.D. dissertation under the supervision of Prof M. N. Daif.

Funding

Not applicable.

Availability of data and materials

Not applicable.

Authors' contributions

Both authors read and approved the final manuscript.

Competing interests

The authors declare that they have no competing interests.

\section{Publisher's Note}

Springer Nature remains neutral with regard to jurisdictional claims in published maps and institutional affiliations.

Received: 18 June 2018 Accepted: 9 September 2018

Published online: 04 April 2019

\section{References}

1. Herstein, I. N.: Jordan derivations of prime rings. Proc. Amer. Math. Soc. 8, 1104-1110 (1957)

2. Brešar, M.: Jordan derivations on semiprime rings. Proc. Amer. Math. Soc. 104, 1003-1006 (1988)

3. Brešar, M.: Jordan mappings of semiprime rings. J. Algebra. 127, 218-228 (1989)

4. Jing, W., Lu, S.: Generalized Jordan derivations on prime rings and standard operator algebras. Taiwan. J. Math. 7, 605-613 (2003)

5. Liu, C., Shiue, W.: Generalized Jordan triple $(\theta, \phi)$-derivations on semiprime rings. Taiwan. J. Math. 11(5), 1397-1406 (2007)

6. Vukman, J., Kosi-Ulbl, I.: On centralizers of semiprime rings. Aequationes Math. 66(3), 277-283 (2003)

7. Daif, M. N., El-Sayiad, M. S., Muthana, N. M.: An identity on $\theta$-centralizers of semiprime rings. Int. Math. Forum. 937-944(3) (2008)

8. Nakajima, A.: Note on generalized Jordan derivation associate with Hochschild 2-cocycles of rings. Turkish J. Math. 30, 403-411 (2006)

9. Nawzad, A., Abdulla, H., Majeed, A. H.: Generalized derivations on semiprime rings. Sci. Magna. 6(3), 34-39 (2010)

10. Rehman, N., Hongan, M.: Generalized Jordan derivations on Lie ideals associate with Hochschild 2-cocycles of rings. Rend. Circolo Matematico Palermo. 60(3), 437-444 (2011)

11. Martindale III, W. S: Prime rings satisfying a generalized polynomial identity. J. Algebra. 12, $576-584$ (1969)

12. Chuang, C.: GPIs having coefficients in Utumi quotient rings. Proc. Amer. Math. Soc. 103, 723-728 (1988)

\section{Submit your manuscript to a SpringerOpen ${ }^{\circ}$ journal and benefit from:}

- Convenient online submission

- Rigorous peer review

- Open access: articles freely available online

- High visibility within the field

- Retaining the copyright to your article

Submit your next manuscript at $\gg$ springeropen.com 